\title{
Pre-hospital time intervals in trauma patient transportation by emergency medical service: association with the first 24- hour mortality
}

Afsaneh Esmaeiliranjbar ${ }^{1}$, Masoud Mayel' ${ }^{1}$, Mitra Movahedi ${ }^{1}$, Faezeh Emaeiliranjbar $^{2}$, Amirhossein Mirafzal ${ }^{*}$

'Department of Emergency Medicine, Kerman University of Medical Sciences, Kerman, Iran

${ }^{2}$ Department of Biology, Kerman Branch of Islamic Azad University, Kerman, Iran

\author{
Received: 2 June 2015 \\ Accepted: 24 June 2015 \\ Published online: 10 October 2015 \\ *Corresponding author: Amirhossein \\ Mirafzal, Department of Emergency \\ Medicine, Kerman University of \\ Medical Sciences, Kerman, Iran. \\ Tel: +989121362984 \\ Email:mirafzal@kmu.ac.ir \\ Competing interests: The authors \\ declare that there are no conflict \\ of interest or support sources for \\ this work. The authors take the \\ responsibility of this declaration. \\ Funding information: None. \\ Citation: Esmaeiliranjbar A, Mayel \\ M, Movahedi M, Emaeiliranjbar F, \\ Mirafzal A . Pre-hospital time intervals \\ in trauma patient transportation \\ by emergency medical service: \\ association with the first 24-hour \\ mortality. Journal of Emergency \\ Practice and Trauma 2016; 2(2): 37-41. \\ doi: $10.15171 /$ jept.2015.15
}

\begin{abstract}
Objective: Most previous retrospective studies failed to show a consistent association between pre-hospital time intervals and mortality in trauma patients, bringing the recommendation of "transport fast to increase survival" under question. The aim of this study was to evaluate the association of response time, scene time, and transport time with 24-hour in-hospital mortality.

Methods: In this cross-sectional study data were collected In the emergency department (ED). Time intervals were obtained from emergency medical service (EMS) central system. All traumatized patients presented to an urban academic hospital by EMS with Emergency Severity Index (ESI) levels 1 or 2 were included in the study. Exclusion criteria were age under 16 or above 65 , being transported from outside of the city, severe underlying medical illness, life threatening intoxications, and randomized trauma score (RTS) of more than 10. Patients were followed in the hospital for 24-hour mortality.

Results: A total of 2884 patients were enrolled in the study. Response time, scene time, transport time, and total out of hospital time were all associated with mortality in univariate analysis $(P=0.02,0.01,<0.001$, and 0.001 , respectively). In multivariate regression analysis, transport time was associated with 24-hour mortality $(P<0.001$, OR [95\% Cl]: 1.20 [1.161.24]).

Conclusion: Although time intervals in most previous studies did not show association with mortality, there is no recommendation such as "pre-hospital time intervals in traumatized patients should not be limited," since limiting time intervals for taking a traumatized patient to the hospital still seems to be prudent. Our findings support the recommendation of decreasing the transportation and total out of hospital time in the present condition in Kerman city EMS system.

Keywords: Prehospital Emergency Care, Time-to-Treatment, Trauma, Transportation, Emergency medical service
\end{abstract}

\section{Introduction}

Direct transportation of critically ill trauma patients should be performed in an organized trauma system and direct transfer to a trauma center in some cases (e.g. severe traumatic brain injury) should not be sacrificed for the concept of "initial stabilization" at a nearer non-trauma center $(1,2)$. In order to transport a trauma patient to a trauma center, the rationale of decreasing the out of hospital time to increase survival is long being considered by authors and emergency medical systems (EMSs) (3). Some authors have considered this variable as a total out of hospital time $(4,5)$, whereas most researchers in the recent years have divided the total out of hospital time to a number of "intervals," including a response time (time from
EMS system activation to presence of an ambulance on the scene), scene (or on-scene) time (time that an ambulance spends on the scene to the beginning of departure), and transport time (time from the beginning of departure to patient's arrival at the emergency department [ED]) (6$8)$. The correlations among varying combinations of these intervals with trauma-related mortality were evaluated by different studies. There are also some studies which assessed these intervals with mortality in non-trauma states, including stroke, sepsis, acute heart failure (AHF), and acute myocardial infarction (AMI) (9-11). Although some multicenter studies with large populations were done regarding time intervals and mortality, nearly all of them are performed in a retrospective manner. In addition, there is 
still no universal recommendation based on these findings, since there is inconsistency among their results according to different systems and settings. In the present study, we have evaluated a relatively large population to find possible associations between pre-hospital time intervals and in-hospital 24-hour mortality to provide further evidence regarding this conflicting issue.

\section{Methods}

This cross-sectional study was performed prospectively on trauma patients presenting by EMS to Bahonar academic hospital, a level I urban trauma center in Kerman, a large city with a population of near 1 million in the southeast of Iran. Bahonar hospital is the main referral center for trauma patients with an annual ED census of nearly 75000 . All measurements in this study were done 24 hours before the outcome happened.

In a period of 17 months (November 20, 2013 to May 20, 2015), trauma patients brought to Bahonar hospital by EMS in the presence of one researcher and triaged to the resuscitation room were considered as eligible patients. Triage in Bahonar hospital is performed by a trained nurse blinded to our study objectives. The researchers ( 2 residents of emergency Medicine -2 constant persons - in post graduate year [PGY] 3) did not interfere with triage. In our center, a 5-level Emergency Severity Index (ESI) (12-14) system is used for triage, with only ESI level 1 or 2 patients are transferred to the resuscitation room. Exclusion criteria were age under 16 or above 65 , being transported from outside the official territory of the city, presence of severe medical illness potentially affecting the study outcomes, severe and life threatening intoxications, and randomized trauma score (RTS) of more than 10 (15). The EMS system in Kerman city is an integrated and wellfunctioning system including 15 all-time stand-by ambulances, 2 ambulance-buses, and 1 helicopter (for outside of the city events). This system does not use motorcycleambulances (motor lances). All ambulance vehicles have 2 EMTs; at least one of them is paramedic. The vehicles do not use automatic electrical defibrillators for resuscitation, and they follow a local pre-hospital life support system (16). Before conducting the study, we did a pilot study with 50 patients divided between the 2 researchers to measure their inter-observer agreement in calculating RTS. The Spearman correlation coefficient was 0.84 , which was in the excellent range. During the study, an attending physician of emergency medicine (a single person) periodically and randomly visited the patients simultaneously with the researchers, assessing the agreements between measurements. Fortunately, the agreements were all in the excellent range during the study. The remaining cases of disagreement were solved by consensus. A convenience sample of patients with trauma who passed the inclusion and exclusion criteria were enrolled in the study. For each patient, 3 time intervals (response time, scene time, and transport time) were obtained according to the records of the EMS computerized central system. The source of time intervals was chosen to be the computerized system because EMS dispatch operator immediately records the times when technicians announce scene arrival, departure, or ED arrival. Other potentially confounding variables recorded were age, sex, and mechanism of injury (falls, motor vehicle collisions [MVCs], etc). After completing the standardized sheet by researchers, all patients were followed during the first 24 hours of admission for mortality- our only outcome.

Values are reported as mean \pm standard deviation (SD) for normally distributed continuous variables. To report variables without a normal distribution, median \pm inter quartile range (IQR) was used. Proportions were presented as percentages with 95\% CI. For univariate analysis, continuous data were analyzed using the Student $t$ test according to the Kolmogorov-Smirnov test (All data had normal distribution) and categorical data were compared using Pearson $\chi 2$ test. In multivariable model, we entered all variables with a potential to predict the outcome according to univariable analysis results. All variables with $P$ values less than 0.25 in the univariable analysis were put into a logistic regression analysis (17) to determine independent relationships with 24 -hour mortality. The multivariable analysis was performed by constructing a logistic regression model using forward Wald method and odds ratios (ORs) for variables were reported. Statistical analysis was performed by SPSS version 16.0 (SPSS Inc., Headquarters, Chicago, IL) software program. A $P$ value less than 0.05 was considered to be statistically significant.

\section{Results}

From an eligible population of 3240 patients triaged to resuscitation room in the study period in the presence of researchers, 2884 patients were enrolled in the study (Figure 1). 1902 (70\%) were males and 982 (30\%) were females. The mean (SD) age was 30.23 (12.4). The mechanism of injury was MVC in 2587 (89\%), street or home fights in 67 $(2.3 \%)$, and fall injury in $364(12.7 \%)$ patients. The mean (SD) values for response time, scene time, transport time, and total out of hospital time were 8.5 (3.6), 12.9 (5.1), 9.8 (4.1), and 31.3 (8.3) minutes, respectively. Minimummaximum values for these variables were 2-26, 2-46, 3-37, and 10-75 minutes, respectively. In the first 24 hours of follow-up, 147 (5\%) patients died attributed to trauma.

Age, sex, and mechanism of injury were not associated with 24 -hour mortality $(P=0.34,0.7$, and 0.26 , respectively), but response time, scene time, transport time, and total out of hospital time showed associations with this outcome in the $t$ test (Table 1).

Because response time, scene time, and transport time have co-linear relations with total out of hospital time, we created the final model excluding the latter variable. In multivariable logistic regression analysis, the model run by entering response time, scene time, and transport time showed an independent association between transport 


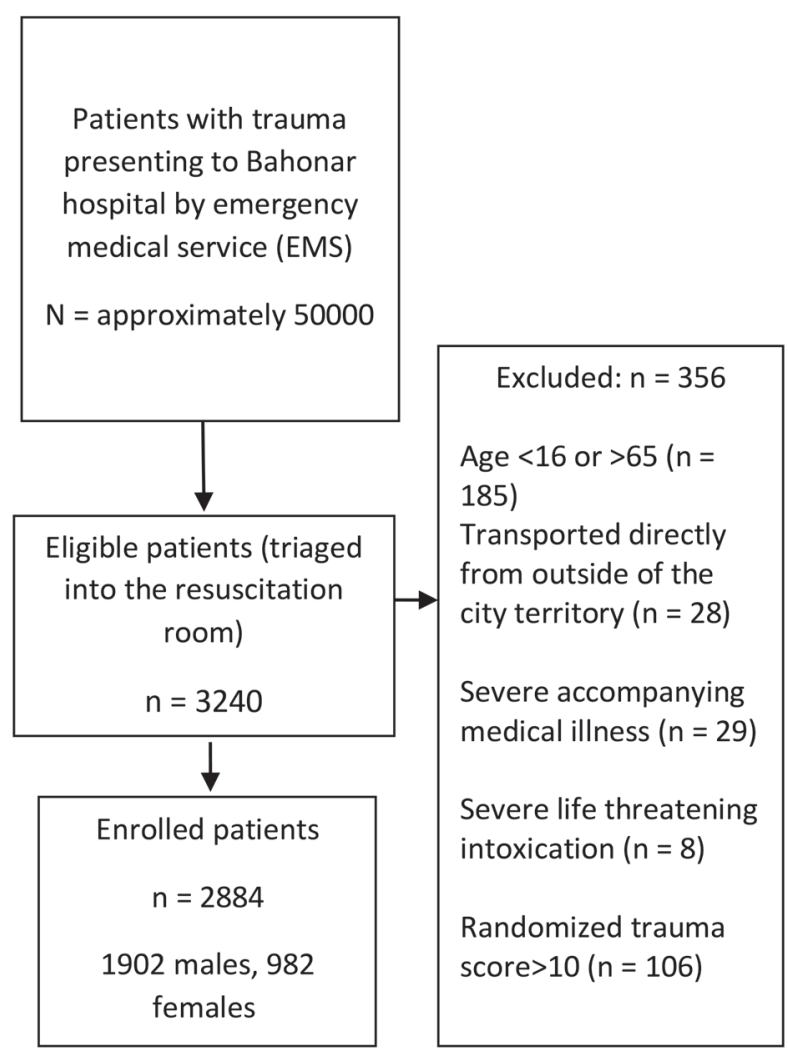

Figure 1. Study population.

Table 1. Variables associated with mortality in univariable analysis

\begin{tabular}{lcc}
\hline Variable & OR $(\mathbf{9 5} \% \mathbf{C I})$ & P value \\
\hline Response time & $0.95(0.90-0.99)$ & 0.02 \\
Scene time & $0.96(0.92-0.99)$ & 0.01 \\
Transport time & $1.17(1.14-1.21)$ & $<0.001$ \\
Total out of hospital time & $1.03(1.01-1.05)$ & 0.001 \\
\hline
\end{tabular}

Table 2. Variables associated with mortality in multivariable logistic regression

\begin{tabular}{llll}
\hline $\begin{array}{l}\text { Variables entered } \\
\text { in the model }\end{array}$ & $\begin{array}{l}\text { Variable showing } \\
\text { association }\end{array}$ & OR $(95 \% \mathrm{Cl})$ & $\boldsymbol{P}$ value \\
\hline $\begin{array}{l}\text { Response time } \\
\begin{array}{l}\text { Scene time } \\
\text { Transport time }\end{array}\end{array}$ & Transport time & $1.20(1.16-1.24)$ & $<0.001$ \\
\hline
\end{tabular}

time and mortality in the final step (Table 2). When dividing patients into 2 groups with total out of hospital times of less than 60 minutes and 60 minutes or more in logistic regression analysis, 21 patients in the latter group showed increased mortality by a factor of 22 relative to the other group.

\section{Discussion}

In the setting of non-traumatic out of hospital cardiac arrest, it was shown that pre-hospital time intervals would make a survival and system performance benefit $(18,19)$, although there are studies which did not find any association between transport time and mortality in this setting and concluded that a modest increase in transport time may be beneficial at the cost of patient's arrival at a highly equipped center (20). In case of trauma patients, the reverse scenario seems to be present: the literature fails to show a consistent association between pre-hospital intervals and mortality, but there are some studies showing benefit from lower time intervals in urban (6) and rural (21) areas. Also, in a large multicenter study performed by Newgard et al (22), a survival benefit was shown in patients with penetrating trauma if the scene time was less than 10 minutes over those with scene times over 20 minutes. Although there is a famous out of hospital "golden hour" in pre-hospital trauma transport conversations, this large study failed to show any difference in mortality between the 2 groups with total out of hospital times of 60 minutes or less and more than 60 minutes. Results of our study showed independent association between transport time with 24-hour mortality, which is inconsistent with previously larger studies. By each minute increase in transport time, there will be a $20 \%$ rise in 24 -hour mortality. However, there are differences between our study and larger retrospective and multicenter studies performed previously: first, data collection in this study (except for time measurements) were done in a prospective manner with pre-programming; second, by choosing RTS more than 10 as an exclusion criteria, we automatically enrolled more ill patients relative to most previous studies; third, we limited our outcome to 24-hour mortality presuming the fact that mortality after this period may be due to more confounding factors; and lastly, this study is performed in a different setting and different EMS system from north America. It can be concluded that recommendations for out of hospital time frame limitations and procedures may be best written with great respect to local circumstances. We have found associations between 24-hour mortality with response time, scene time, and total out of hospital time in univariate analysis. The OR of total out of hospital time showed that when total out of hospital time increases by 1 minute, the 24 -hour mortality rate will rise by $3 \%$. However, we did not enter the total out of hospital time in the final model because there is close correlation with this variable and other time intervals. It was optimal that we were able to measure time intervals ourselves, like the other variables, but this was impractical according to our resources. Also, we included and excluded patients and measured RTS in the time of ED entrance, which were several minutes later than the event. We could not measure vital signs by ourselves in the field, but we preferred not to rely on field recorded vital signs, since our experience implied exact measurements cannot be done in the field conditions. This, however, can act as a source of bias in our study. We also did not measure injury severity score (ISS) (ISS measurement is not a routine practice among the emergency physicians in Iran and most of them use RTS instead) for patients and this anatomic trauma score 
may also act as a confounding factor. The major limitation of our study was its restriction to one large city with a smaller study population relative to previous larger and multicenter studies. Therefore, our results could not be extrapolated to other regions easily.

\section{Conclusion}

Transport time showed a significant independent association with in-hospital 24-hour mortality in our study. It seems that local circumstances play a significant role in our findings, since previous larger studies in the developed world failed to show such an association. Although time intervals in most previous studies did not show association with mortality, there is no recommendation such as "pre-hospital time intervals in traumatized patients should not be limited," since limiting time intervals for taking a traumatized patient to the hospital still seems to be prudent. Our findings support the recommendation of decreasing the transportation and total out of hospital time in the present condition in Kerman city EMS system.

\section{Ethical issues}

This study was approved by the Institutional Review Board and ethics committee of Kerman University of Medical Sciences.

\section{Authors' contributions}

AER conceived the study, collected the data, and participated in drafting the manuscript. AM conceived the study, supervised data analysis, drafted the manuscript, and revised the final version. MaMa conceived the study, supervised data collection process, supervised the drafting, and revised the final version of the manuscript. MiMo collected the data, participated in data analysis, and participated in drafting of the manuscript. FER performed the data analysis, drafted the results section, and supervised the final revision of the manuscript.

\section{References}

1. Hartl R, Gerber LM, Iacono L, Ni Q, Lyons K, Ghajar J. Direct transport within an organized state trauma system reduces mortality in patients with severe traumatic brain injury. J Trauma 2006; 60(6): 1250-6.

2. Nirula R, Maier R, Moore E, Sperry J, Gentillelo L. Scoop and run to the trauma center or stay and play at the local hospital: hospital transfer's effect on mortality. J Trauma 2010; 69(3): 595-9. doi: 10.1097/ TA.0b013e3181ee6e32.

3. Lerner EB, Moscati RM. The golden hour: scientific fact or medical "urban legend"? Acad Emerg Med 2001; 8(7): 758-60.

4. Petri RW, Dyer A, Lumpkin J. The effect of prehospital transport time on the mortality from trauma patients. Prehosp Disaster Med 1995; 10(1): 24-9.

5. Pepe PE, Wyatt CH, Bickel WH, Bailey ML, Mattox KL. The relationship between total pre-hospital time and outcome in hypotensive victims of penetrating injuries. Ann Emerg Med 1987; 16(3): 293-7.

6. Feero S, Hedges JR, Simmons E, Irwin L. Does out of hospital EMS time affect trauma survival? Am J Emerg Med 1995; 13(2): 133-5.

7. Carr BG, Caplan JM, Pryor JP, Branas CC. A metaanalysis of pre-hospital care times for trauma. Prehosp Emerg Care 2006; 10(2): 198-206.

8. Sloan EP, Callahan EP, Duda J, Sheaf CM, Robin AP, Barret JA. The effect of urban trauma system hospital bypass on pre-hospital transport times and level 1 trauma patient survival. Ann Emerg Med 1989; 18(11): 1146-50.

9. Ramanujam P, Castillo E, Patel E, Vilke G, Wilson MP, Dunford JV. Prehospital transport time intervals for acute stroke patients. J Emerg Med 2009; 37(1): 40-5. doi: 10.1016/j.jemermed.2007.11.092.

10. Seymour CW, Rea TD, Kahn JM, Walky AJ, Yealy DM, Angus DC. Severe sepsis in pre-hospital emergency care: Analysis of incidence, care, and outcome. Am J Respir Crit Care Med 2012; 186(12): 1264-71. doi: 10.1164/rccm.201204-0713OC.

11. Takahashi M, Kohsaka S, Miyata H, Yoshikawa T, Takagi A, Harada K, et al. Association between prehospital time intervals and shor term outcome in acute heart failure patients. J Card Fail 2011; 17(9): 742-7. doi: 10.1016/j.cardfail.2011.05.005.

12. Gilboy N, Tanabe P, Travers D, Rosenau AM. Emergency Severity Index (ESI): a triage tool for emergency department care, version 4 . Implementation handbook 2012 edition. USA: Agency for Healthcare Research and Quality; 2011. http://www.ahrq.gov/sites/default/files/wysiwyg/ professionals/systems/hospital/esi/esihandbk.pdf.

13. Barfod C, Lauritzen MM, Danker JK, Sölétormos G, Forberg JL, Berlac PA, et al. Abnormal vital signs are strong predictors for intensive care unit admission and in-hospital mortality in adults triaged in the emergency department - a prospective cohort study. Scand J Trauma Resusc Emerg Med 2012; 20: 28. doi: 10.1186/1757-7241-20-28.

14. Garbez R, Carrieri-Kohlman V, Stotts N, Chan G, Neighbor M. Factors influencing patients assignment to level 2 and level 3 within the 5 level ESI triage system. J Emerg Nurs 2011; 37(6): 526-32. doi: 10.1016/j.jen.2010.07.010

15. Chawda MN, Hildebrand F, Pape HC, Giannoudis PV. Predicting outcome after multiple trauma: which scoring system? Injury 2004; 35(4): 347-58.

16. Zargar M, Kalantar Motamedi SM, Karbakhsh M, Ghodsi SM, Rahimi-Movaghar V, Panahi F, et al. Trauma care system in Iran. Chin J Traumatol 2011; 14(3): 131-6.

17. Bursac Z, Gauss CH, Williams DK, Hosmer DW. Purposeful selection of variables in logistic regression. Source Code Biol Med 2008; 3: 17. doi: 10.1186/1751- 
0473-3-17.

18. De Maio VJ, Stiell IG, Wells GA, Spaite DW. Optimal defibrillation response intervals for maximum outof-hospital cardiac arrest survival rates. Ann Emerg Med 2003; 42(2): 242-50.

19. Studnek JR, Garvey L, Blackwell T, Vandeventer S, Ward SR. Association between prehospital time intervals and ST elevation myocardial infarction system performance. Circulation 2010; 122(15): 14649. doi: 10.1161/CIRCULATIONAHA.109.931154.

20. Spaite DW, Bobrow BJ, Vadeboncoeur TJ, Chikani V, Clark L, Mullins T, et al. The impact of prehospital transport interval on survival in out of hospital cardiac arrest: Implications for regionalization of post-resuscitation care. Resuscitation 2008; 79(1): 616. doi: 10.1016/j.resuscitation.2008.05.006.

21. Gonzalez RP, Cummings GR, Phelan HA, Mulekar MS, Rodning CB. Does increased emergency medical services prehospital time affect patient mortality in rural motor vehicle crashes? A statewide analysis. Am J Surg 2009; 197(1): 30-4. doi: 10.1016/j. amjsurg.2007.11.018.

22. Newgard CD, Schmicker RH, Hedges JR, Tricket JP, Davis DP, Bulger EM, et al. Emergency medical services and intervals in trauma: assessment of a "golden hour" in a north American prospective cohort. Ann Emerg Med 2010; 55(3): 235-46. doi: doi: 10.1016/j.annemergmed.2009.07.024. 